\title{
Natural material-decorated mesoporous silica nanoparticle container for multifunctional membrane-controlled targeted drug delivery
}

This article was published in the following Dove Press journal:

International Journal of Nanomedicine

Number of times this article has been viewed

\author{
Yan $\mathrm{Hu}^{\prime}$ \\ Lei $\mathrm{Ke}^{2}$ \\ Hao Chen' \\ Ma Zhuo' \\ Xinzhou Yang' \\ Dan Zhao' \\ Suying Zeng' \\ Xincai Xiao' \\ 'Department of Pharmaceutics, School \\ of Pharmaceutical Science, South- \\ Central University for Nationalities, \\ ${ }^{2}$ Department of Medicinal Chemistry, \\ School of Pharmacy, Tongji Medical \\ College, Huazhong University of \\ Science and Technology, Wuhan, \\ People's Republic of China
}

Correspondence: Yan Hu; Xincai Xiao School of Pharmaceutical Science, South-Central University for Nationalities, 182 Minyuan Road, Wuhan 430074, People's Republic of China Tel +86 I89 429। 55।7;

+86276784 II96

Email huyan200277@I63.com;

xcxiao@mail.scuec.edu.cn
Abstract: To avoid the side effects caused by nonspecific targeting, premature release, weak selectivity, and poor therapeutic efficacy of current nanoparticle-based systems used for drug delivery, we fabricated natural material-decorated nanoparticles as a multifunctional, membrane-controlled targeted drug delivery system. The nanocomposite material coated with a membrane was biocompatible and integrated both specific tumor targeting and responsiveness to stimulation, which improved transmission efficacy and controlled drug release. Mesoporous silica nanoparticles (MSNs), which are known for their biocompatibility and high drug-loading capacity, were selected as a model drug container and carrier. The membrane was established by the polyelectrolyte composite method from chitosan (CS) which was sensitive to the acidic tumor microenvironment, folic acid-modified CS which recognizes the folate receptor expressed on the tumor cell surface, and a $\mathrm{CD}_{44}$ receptor-targeted polysaccharide hyaluronic acid. We characterized the structure of the nanocomposite as well as the drug release behavior under the control of the $\mathrm{pH}$-sensitive membrane switch and evaluated the antitumor efficacy of the system in vitro. Our results provide a basis for the design and fabrication of novel membranecontrolled nanoparticles with improved tumor-targeting therapy.

Keywords: multifunctional, membrane-controlled, natural materials, mesoporous silica nanoparticles, targeted drug delivery

\section{Introduction}

The majority of existing antitumor chemotherapeutic agents have toxic effects on normal cells, leading to undesirable side effects. ${ }^{1}$ An ideal drug delivery system accurately releases the drug at a precise time and location, thereby reducing the possibility of premature or off-target effects. ${ }^{2}$ Despite recent advances in nanoparticle drug delivery systems, achieving an optimal therapeutic effect remains a significant challenge. ${ }^{3-5}$ An advantage of nanoparticle carriers such as mesoporous silica nanoparticles (MSNs), ${ }^{6-12}$ liposomes, ${ }^{13-15}$ and carbon nanotubes, ${ }^{16}$ which are usually considered to be the drug storage "warehouse" for drug delivery, is their high drug-loading (DL) capacity. However, there is a need to increase the stability and biocompatibility of these carriers and improve their target specificity for effectively accurate release at fixed time and location, to avoid off-target or leakage release. ${ }^{17,18}$

Most existing nanoparticle carriers lack the capacity for both tumor targeting and controlled release. Moreover, their preparation is a complicated process using costly raw materials; they can also exhibit poor biocompatibility or even toxicity. Surface modification of nanoparticles can improve their safety and efficacy in drug delivery systems. 
Currently, the main idea of the intelligent design of drug delivery system is controlling the drug release based on the tumor surface receptor targeting and physiological environment. ${ }^{19}$ Natural materials have unique advantages for drug delivery applications since they are not limited in terms of availability, technology required for preparation, amenability to target modification, or cost and are biocompatible, biodegradable, and safe. In addition, many natural materials exhibit unique physiological characteristics. For example, chitosan (CS) dissolves at low $\mathrm{pH}$, which can be exploited for acid-sensitive drug release in the acidic tumor microenvironment. ${ }^{20}$ Moreover, both the $-\mathrm{OH}$ and $-\mathrm{NH}_{2}$ groups in the CS molecule are easily modified for increased functionality. In addition to its moisturizing properties, hyaluronic acid (HA) and its endogenous receptor cluster of differentiation $\mathrm{CD}_{44}$, a transmembrane glycoprotein, have been linked to tumor growth, invasion, and metastasis. ${ }^{21}$ Drug delivery systems have been developed to combine the specificity of the $\mathrm{HA} / \mathrm{CD}_{44}$ interaction with their respective carrier and targeting functions. ${ }^{22,23} \mathrm{MSN}$ has good potential as a carrier for drug delivery due to its mesoporous structure, high specific surface area, biocompatibility, and high DL capacity. ${ }^{24-29}$

Therefore, the combination of natural materials and a nanoparticle carrier first solves the problem of the biocompatibility of the drug delivery system, and second the excellent physiological response and modification of the natural materials lay the foundation for realizing the multifunctional transportation of the drug delivery system and accurate drug release at a fixed time and location. In this study, we designed a multifunctional membrane-controlled MSN-based drug delivery system that exploits the physiological responsiveness of natural materials. We evaluated its capacity for drug delivery and antitumor efficacy in vitro. Our results demonstrate that this system can improve the efficacy of cancer chemotherapy.

\section{Materials and methods Materials}

HA (molecular weight [MW]: $3.3 \times 10^{5}$ ) was obtained from Freda Biological Technology Co., Ltd (Shandong, People's Republic of China). CS (MW: $1 \times 10^{6}$ ) with a deacetylation degree of $95 \%$ was obtained from Jinke Biochemistry Co., Ltd (Zhejiang, People's Republic of China). Cetyltrimethylammonium bromide (CTAB), tetraethyl orthosilicate (TEOS), ethylene glycol, and succinic anhydride were purchased from Sinopharm Chemical Reagent Co., Ltd (Shanghai, People's Republic of China). 3-(Aminopropyl) trimethoxysilane, doxorubicin (DOX), folic acid (FA), 1-ethyl-3-[3-(dimethylamino)propyl] carbodiimide (EDC), and $N$-hydroxysuccinimide (NHS) were purchased from Aladdin Reagent Co., Ltd (Shanghai, People's Republic of China). Dulbecco's Modified Eagle's Medium (DMEM), fetal bovine serum (FBS), trypsase, penicillin-streptomycin 3-(4,5-Dimethylthiazol-2-yl)-2, 5-diphenyltetrazolium bromide (MTT) and Hoechst33258 were purchased from Sigma-Aldrich Co (St Louis, MO, USA). All the other reagents were of analytical grade. HepG2 cells and L02 cells came from Shanghai Institutes for Biological Sciences of the Chinese Academy of Sciences (Shanghai, People's Republic of China).

\section{MSN synthesis}

MSNs were prepared by adapting a previously described sol-gel method. ${ }^{30-32}$ Briefly, $1.2 \mathrm{~g} N$-CTAB was dissolved in ultrapure water at $60^{\circ} \mathrm{C}$ with vigorous stirring. A $30 \mathrm{~mL}$ volume of ethylene glycol and $7.2 \mathrm{~mL}$ ammonia solution (25\%-28\%) were added with stirring until the solution was clear; $2.4 \mathrm{~mL}$ TEOS was rapidly added, and the mixture was stirred for $2 \mathrm{~h}$ at $60^{\circ} \mathrm{C}$ and then stored at $60^{\circ} \mathrm{C}$ for $24 \mathrm{~h}$ followed by storage at $4^{\circ} \mathrm{C}$ overnight. After washing three times with ethanol, the product was vacuum dried at $50^{\circ} \mathrm{C}$. To remove the surfactant, the as-synthesized product was refluxed in $54.30 \mathrm{~mL}$ absolute ethanol with $0.54 \mathrm{~mL}$ hydrochloric acid $(36 \%-38 \%)$ at $80^{\circ} \mathrm{C}$ for $6 \mathrm{~h}$; the process was repeated six times. The final product was collected by centrifugation, washed with ethanol followed by water four times, and dried at $50^{\circ} \mathrm{C}$ under vacuum.

\section{Amino-functionalized MSN synthesis and carboxylation}

The surface of MSNs was functionalized with amine groups $\left(-\mathrm{NH}_{2}\right)$ by treatment with 3-aminopropyl trimethoxysilane (APTS). ${ }^{32,33}$ The as-synthesized MSNs (0.2 g) were dispersed in anhydrous toluene $(20 \mathrm{~mL})$ containing APTS $(0.40 \mathrm{~mL})$ under vigorous stirring. The mixture was gently stirred for $24 \mathrm{~h}$ at $120^{\circ} \mathrm{C}$. The product $\left(\mathrm{MSN}-\mathrm{NH}_{2}\right)$ was centrifuged and washed with ultrapure water and methanol three times, and then vacuum dried at $50^{\circ} \mathrm{C}$. The $\mathrm{MSN}_{-} \mathrm{NH}_{2}(0.2 \mathrm{~g})$ was dispersed in acetone $(20 \mathrm{~mL})$ under ultrasound with vigorous stirring for $4 \mathrm{~h}$. Acetone $(20 \mathrm{~mL})$ containing succinic anhydride (3 g) was added to the solution with stirring for another $24 \mathrm{~h}$. The resultant powder (MSN-COOH) was filtered and rinsed with ultrapure water and ethanol three times to remove residual acidic compounds. ${ }^{33}$ 


\section{Synthesis of FA-modified CS (FA-CS)}

FA (74 mg; $0.167 \mathrm{mmol}$ ) was added to $29.5 \mathrm{~mL}$ dimethyl sulfoxide (DMSO) and dissolved thoroughly at room temperature. Then, $322 \mathrm{mg}$ EDC $(1.68 \mathrm{mmol})$ and $190 \mathrm{mg}$ NHS $(1.68 \mathrm{mmol})$ were added at room temperature in the dark and stirred for $1 \mathrm{~h}$ to obtain the red-brown FA-activated ester DMSO solution. ${ }^{34} \mathrm{CS}(29.7 \mathrm{mg})$ was dissolved in $7.5 \mathrm{~mL}$ acetic acid-sodium acetate buffer ( $\mathrm{pH} 7.4)$, and then the FA-active ester in DMSO solution was added with magnetic stirring for $24 \mathrm{~h}$ in the dark at room temperature. After the reaction, $0.1 \mathrm{~mol} / \mathrm{L} \mathrm{NaOH}$ solution was added to the mixture, and the $\mathrm{pH}$ was adjusted to 9 . The mixture was dialyzed for 3 days in phosphate-buffered saline (PBS; $\mathrm{pH}$ 7.4) to remove the phosphoric acid salt, and then in ultrapure water for 3 more days. Finally, FA-CS was obtained by freeze-drying. To measure the FA content in the FA-CS, the prepared FA-CS was dissolved in 1\% acetic acid solution and prepared to a certain concentration. According to the characteristic absorption of FA at $363 \mathrm{~nm}$, a standard curve was used to calculate the FA content in FA-CS. FA coupling ratio $=$ (the amount of FA in the FA-CS/the amount of FA used) $\times 100 \%$. According to this equation, the FA coupling ratio of FA-CS was calculated to be $27.0 \%$.

\section{Drug loading and capping with functional membrane}

Due to its water solubility and fluorescence properties, DOX was selected as the model drug to evaluate the drug delivery behavior of the carrier. DOX $(1 \mathrm{mg} / \mathrm{mL})$ was prepared in PBS (7.4), ${ }^{35}$ and the nanoparticles (MSN, MSN-NH ${ }_{2}$, or MSN$\mathrm{COOH}$ ) were dispersed in the DOX aqueous solution. After stirring in the dark for $24 \mathrm{~h}$, the drug-loaded nanoparticles were collected by centrifugation, rinsed twice with PBS, and then freeze-dried. The liquid supernatants were combined and determined to calculate DL and entrapment efficiencies.

Composite functional membranes were prepared by the polyelectrolyte composite method and layer-by-layer (LbL) self-assembly. ${ }^{36-38}$ The nanoparticles (MSN, MSN-NH ${ }_{2}$, or MSN-COOH) were resuspended in $10 \mathrm{~mL}$ ultrapure water $(1 \mathrm{mg} / \mathrm{mL})$. The first layer was deposited by adding $5 \mathrm{~mL}$ of $\mathrm{CS}(\mathrm{pH} 3.5,1 \mathrm{mg} / \mathrm{mL})$ or HA $(1 \mathrm{mg} / \mathrm{mL})$ to the aforementioned solution. The mixture was incubated by gentle shaking to allow the adsorption of CS onto the negatively charged MSN (or MSN-COOH) or of HA onto the positively charged MSN-NH $\mathrm{N}_{2}$. Excess CS or HA was removed by three rounds of centrifugation (18,000 rpm, $10 \mathrm{~min}$ ) followed by washing and re-dispersal in water. The subsequent layers were prepared in the same manner. The two polyelectrolytes were alternately deposited until the desired number of coating layers was reached. The polyelectrolyte multilayers of CS and HA were deposited onto the DOX-loaded MSN or MSN derivatives by the same method. The membrane-controlled nanoparticles with three layers and surface layer covered with HA were labeled as DOX@MSN-NH 2 (HA/CS/HA); and nanoparticles with four layers and a surface layer covered by FA-CS were labeled as DOX@MSN-NH 2 (HA/CS/HA/FA-CS).

\section{Characterization of MSN, MSN derivatives, and membrane-coated MSN}

The diameter, distribution, and dispersity of the MSNs were evaluated by dynamic light scattering (DLS). MSNs $(0.1 \mathrm{mg} / \mathrm{mL})$ were dispersed ultrasonically in ultrapure water. After dilution in the detection pool, three parallel measurements were performed for each sample and the average value was calculated. The surface morphology of MSNs was examined by scanning electron microscopy (SEM; JEOL, Tokyo, Japan) after coating a small amount of sample ( $0.2 \mathrm{~g})$ with gold. MSN and membrane-coated MSN morphology was examined by transmission electron microscopy (TEM). Formvar was used to support the copper net; the sample dispersed in ultrapure water $(0.1 \mathrm{mg} / \mathrm{mL})$ was added to the copper net and dried before detection.

The small-angle X-ray diffraction (XRD) pattern of the dried MSN sample was determined on a D8 Advance instrument (Bruker Optik GmbH, Ettlingen, Germany). The tube voltage and current were $40 \mathrm{kV}$ and $40 \mathrm{~mA}$, respectively. $\mathrm{N}_{2}$ adsorption was measured on a fully automatic physicochemical adsorption analyzer (Autosorb-1C-Tcd-Mass; Quantachrome Instruments, Boynton Beach, FL, USA).

The chemical structure of MSNs, modified MSNs, and FA-CS was characterized by infrared (IR) spectroscopy. The sample was mixed with $\mathrm{KBr}$ powder and pressed into tablets. The spectra were measured on a Fourier transform (FT)-IR spectrometer (Spectrum One; PerkinElmer Inc., Waltham, MA, USA) in the wavelength range of $400-4,000 \mathrm{~cm}^{-1}$ with a resolution of $4 \mathrm{~cm}^{-1}$.

The stability of MSNs, modified MSNs, and membranecoated MSNs dispersed in ultrapure water $(0.1 \mathrm{mg} / \mathrm{mL})$ was determined using a potentiometer (Malvern Instruments, Malvern, UK). The zeta potential of the membrane-decorated nanocomposites in different physiological conditions (such as saline solution at $\mathrm{pH} 7.4$ and PBS solution) was also determined. Thermogravimetric (TG) analysis of MSNs and the modified MSNs (5 mg) (TG 209F3; Netzsch, Selb, Germany) was carried out at a heating rate of $10^{\circ} \mathrm{C} / \mathrm{min}$ from $40^{\circ} \mathrm{C}$ to $700^{\circ} \mathrm{C}$ under an $\mathrm{N}_{2}$ atmosphere. 


\section{Analysis of in vitro drug release}

DOX@MSN,DOX@MSN-NH 2 , DOX@MSN-COOH, and DOX@MSN-NH $\mathrm{N}_{2}(\mathrm{HA} / \mathrm{CS} / \mathrm{HA} / \mathrm{FA}-\mathrm{CS})(5 \mathrm{mg})$ were dispersed in $1 \mathrm{~mL}$ PBS ( $\mathrm{pH}$ 7.4) or acetic acid-sodium acetic buffer solution ( $\mathrm{pH}$ 5.0) and then transferred to a dialysis tube (MW cutoff $=14,000$ ) that was immersed in a centrifuge tube containing $5 \mathrm{~mL}$ PBS or acetic acid-sodium acetic buffer solution, respectively, at $37^{\circ} \mathrm{C}$ with shaking at $100 \mathrm{rpm}$ under light-sealed conditions. At specific times $(0.5,1.5,3.5,5.5$, $8.5,11.5,24,36$, and $48 \mathrm{~h}$ ), the release medium was replaced with an equivalent volume of fresh release medium. DOX content in the release medium was determined by ultraviolet-visible light (UV-Vis) spectrophotometry (Lambda 35; PerkinElmer Inc.) at a wavelength of $483 \mathrm{~nm}$ and was calculated with a standard curve equation. The DL efficiency and encapsulation efficiency (EE) of the nanoparticles were determined using the following equations:

$$
\begin{aligned}
& \text { DL } \%=\frac{\text { DOX in feed }- \text { free DOX }}{\text { Nanoparticles in feed }} \times 100 \% \\
& \text { EE } \%=\frac{\text { DOX in feed }- \text { free DOX }}{\text { DOX in feed }} \times 100 \%
\end{aligned}
$$

The cumulative release of DOX was calculated using the following equation:

$$
\text { Cumulative release }(\%)=\frac{\mathrm{V}_{0} \mathrm{C}_{\mathrm{n}}+\mathrm{V} \sum \mathrm{C}_{(\mathrm{n}-1)}}{\mathrm{w}} \times 100 \%(
$$

where $\mathrm{C}_{\mathrm{n}}$ and $\mathrm{C}_{(\mathrm{n}-1)}$ are the DOX concentrations for samples $\mathrm{n}$ and $\mathrm{n}-1$, respectively; $\mathrm{V}_{0}$ is the initial volume of the drug release medium; $\mathrm{V}$ is the sampling volume; and $\mathrm{w}$ is the mass of drug loaded.

\section{Cell culture and cell uptake experiments}

Human normal liver (L02) and liver tumor (HepG2) cells were seeded in DMEM containing 10\% heat-inactivated FBS, $100 \mathrm{U} / \mathrm{mL}$ penicillin, and $100 \mu \mathrm{g} / \mathrm{mL}$ streptomycin and cultured in a humidified incubator at $37^{\circ} \mathrm{C}$ and $5 \% \mathrm{CO}_{2}$. The culture medium was changed every 2 days.

Cellular uptake and intracellular release behaviors of DOX-loaded nanoparticles in HepG2 cells were evaluated by fluorescence microscopy on an IX-51 inverted microscope equipped with a $100 \mathrm{~W}$ mercury-xenon arc lamp as an excitation light source and high-speed charge-coupled device camera (Olympus Corporation, Tokyo, Japan). HepG2 cells seeded in six-well plates at a density of $1 \times 10^{5} /$ well were incubated with free DOX and DOX-loaded composite nanoparticles (final DOX concentration: $10 \mu \mathrm{g} / \mathrm{mL}$ ) at $37^{\circ} \mathrm{C}$ for $2 \mathrm{~h}$. After fixing with 4\% paraformaldehyde solution for $30 \mathrm{~min}$, cells were washed three times with PBS, and nuclei were stained with $10 \mu \mathrm{g} / \mathrm{mL}$ Hoechst 33258 in DMEM for $15 \mathrm{~min}$. The culture supernatant was removed, and cells were rinsed twice with PBS prior to observation.

The role of $\mathrm{FA}$ and $\mathrm{CD}_{44}$ receptors in receptor-mediated uptake was investigated by confocal laser scanning microscopy (CLSM; LSM 700; Carl Zeiss Meditec AG, Jena, Germany) according to the procedures reported in the literature.$^{39}$ HepG2 cells in logarithmic growth phase were digested with $0.25 \%$ trypsin, and $5 \times 10^{4}$ cells/well were seeded in a 12-well plate in culture medium without or with $\mathrm{FA}(1 \mathrm{mM})$. After incubation at $37^{\circ} \mathrm{C}$ in $5 \% \mathrm{CO}_{2}$ for $24 \mathrm{~h}$, the DOX@MSN-NH 2 (HA/CS/HA/FA-CS) carrier (final DOX concentration: $10 \mu \mathrm{g} / \mathrm{mL}$ ) was added, followed by incubation for $2 \mathrm{~h}$. The medium was discarded, and cells were washed three times with PBS to remove dead cells and remaining nanoparticles before observation by CLSM. In the same manner, cell culture medium without or with $\mathrm{HA}(2 \mathrm{mg} / \mathrm{mL})$ was used to examine the role of $\mathrm{HA}$ and $\mathrm{CD}_{44}$ receptors on the cellular uptake of DOX@MSN-NH $2(\mathrm{HA} / \mathrm{CS} / \mathrm{HA})$ by CLSM.

\section{In vitro cytotoxicity assay}

As reported in our previous study, drug delivery materials based on MSN are more toxic to HepG2 cells than to normal cells. ${ }^{40}$ Therefore, in this study, we evaluated the cytotoxicity of empty (without DOX) MSN, MSN-COOH, and MSN-NH suspensions in L02 cells with MTT assay to determine their safety as drug carriers. L02 cells in logarithmic phase were digested with $0.25 \%$ trypsase and then seeded in 96-well plates at a density of $1 \times 10^{5} /$ well and cultured at $37^{\circ} \mathrm{C}$ and $5 \% \mathrm{CO}_{2}$ for $24 \mathrm{~h}$. MSN, MSN-COOH, and $\mathrm{MSN}^{-\mathrm{NH}_{2}}$ suspensions $(200 \mu \mathrm{L})$ with concentrations of $0.001,0.005,0.01$, $0.05,0.1,0.5,1.0,5.0,8.0$, and $10 \mu \mathrm{g} / \mathrm{mL}$ were added to cells, with five replicates prepared for each treatment group. Cells were cultured for $24 \mathrm{~h}$, and the culture supernatant was removed; MTT was added to each well followed by incubation for $4 \mathrm{~h}$. A $150 \mu \mathrm{L}$ volume of dimethylsulfoxide was added to dissolve the formazan crystals, and the absorbance at $572 \mathrm{~nm}$ was measured on a microplate reader. Cell viability was calculated using the formula: $\%$ viability $=\mathrm{A}_{\mathrm{s}} /$ $A_{c}$, where $A_{s}$ and $A_{c}$ are the absorbance of the treated and untreated samples, respectively. The same method was used to determine the cytotoxicity of free DOX and DOX-loaded nanoparticles (DOX@MSN, DOX@MSN-NH, DOX@ 
MSN-COOH, DOX@MSN-NH 2 [HA/CS/HA]) at various concentrations (final DOX concentration: 0.1, 0.5, 1, 5, and $10 \mu \mathrm{g} / \mathrm{mL}$ ) in L02 and HepG2 cells.

\section{Statistical analysis}

All the experiments were performed at least three times, and the acquired results are shown as mean \pm SD. The statistical analysis was analyzed by using Origin 8.0 software (OriginLab Corporation, Northampton, MA, USA) via one-way analysis of variance (ANOVA) and Student's $t$-test.

\section{Results and discussion Construction of multifunctional membrane-controlled nanoparticles}

We constructed an MSN-based drug delivery system in which the MSN surface was covered with a functional membrane by the polyelectrolyte composite method or LbL self-assembly (Figure 1). Drug release was controlled by the composite membrane switch, which consisted of the acidsensitive materials CS, FA receptor-targeted CS derivative (FA-CS), and the $\mathrm{CD}_{44}$ receptor-targeted polysaccharide HA. The polyanion HA and polycation CS polymers alternately covered the MSN surface. The outer layer was coated with HA or CS-FA, which targeted the nanoparticles to the $\mathrm{CD}_{44}$ or FA receptor, respectively. Thus, a multifunctional membrane-controlled nanocomposite carrier was constructed
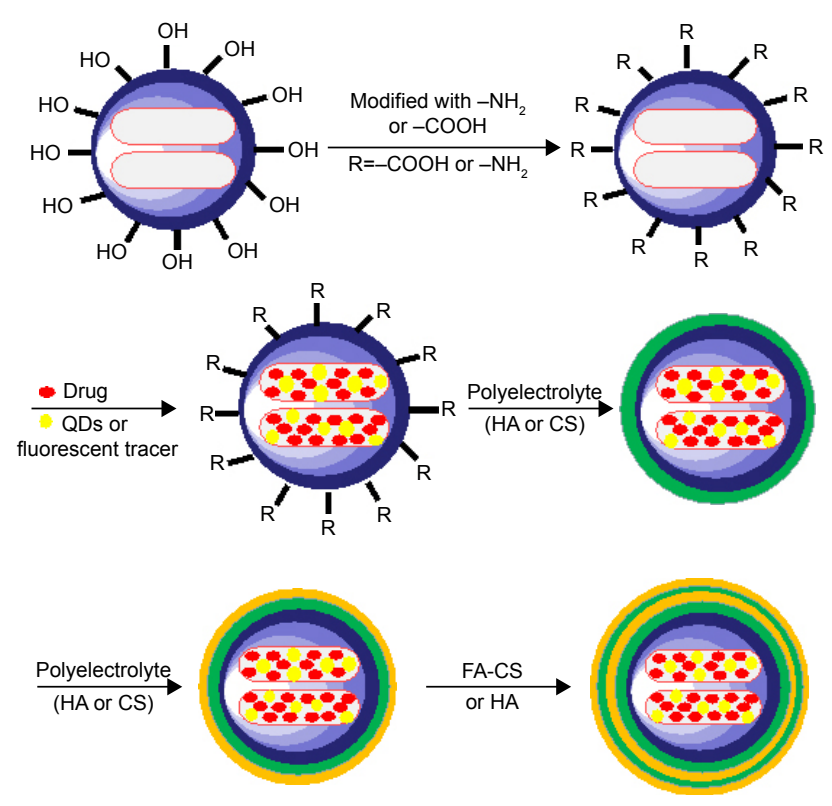

Figure I Fabrication mechanisms of multifunctional membrane-controlled MSNs with drugs and fluorescent tracer.

Abbreviations: CS, chitosan; FA, folic acid; FA-CS, FA-modified CS; HA, hyaluronic acid; MSN, mesoporous silica nanoparticle; QD, quantum dot. that targeted tumor surface receptors and was $\mathrm{pH}$ responsive to enhance the efficacy of the anticancer drug delivery and reduce the cytotoxicity to normal cells.

\section{Physicochemical characterization of MSN, MSN derivatives, and membrane- decorated MSN}

MSN was synthesized according to the method outlined in Figure 2A. To obtain insight into the morphology and structural properties of MSN, SEM, TEM, $\mathrm{N}_{2}$ adsorption analysis, and small-angle XRD analysis were used. The MSNs had a spherical porous structure (Figure 2B). TEM analysis confirmed that MSNs were uniform (Figure 2C); their size was approximately $120 \mathrm{~nm}$, making them suitable for drug delivery. Consistent with this observation, DLS analysis showed that the particle size was $125 \mathrm{~nm}$ and the polydispersity index (PDI) was $<0.2$ (Figure 2D). These results indicate that MSNs had a solid and rigid structure that did not deform in water. The $\mathrm{N}_{2}$ adsorption analysis (Figure $3 \mathrm{~A}$ and $\mathrm{B}$ ), and small-angle XRD pattern (in which the curve shows a wide peak at approximately $2 \theta=2.1^{\circ}$; Figure $3 \mathrm{C}$ ), indicated that MSNs had uniform mesoporous channels and a relatively narrow pore size distribution. The specific surface area of the MSNs was $540.5 \mathrm{~m}^{2} / \mathrm{g}$ (Figure 3A), and the pore size was $8.19 \mathrm{~nm}$ (Figure 3B), which can facilitate drug loading.

The synthesis of MSN-NH $\mathrm{N}_{2}$ and MSN-COOH was performed as shown in Figure 2A. The physicochemical characterization of MSN derivatives included IR spectra, zeta potential, and thermal gravimetric analyses, as shown in Figure 4. The IR spectra revealed peaks at 1,639 and $1,540 \mathrm{~cm}^{-1}$ (Figure 4A) that were attributed to stretch vibrations of amide I and flexural vibrations of the amino group, respectively, indicating that the amino modification was successful. The peaks near 1,711 and $1,418 \mathrm{~cm}^{-1}$ were assigned to symmetric and asymmetric $-\mathrm{COO}^{-}$stretching vibrations, indicating that the MSN structure was modified with a carboxyl group. ${ }^{33}$ Although there was no obvious difference in average diameter between MSN and its derivative (Figure 4B and Table S1), the negative potential of MSN $(-25 \pm 0.75 \mathrm{mV})$ was changed to a positive potential $(+16.7 \pm 0.42 \mathrm{mV}$; Figure $4 \mathrm{~B})$, confirming that the MSN surface was modified with an amino group. Meanwhile, the positive potential of $\mathrm{MSN}-\mathrm{NH}_{2}$ was changed to a negative potential $(-29.6 \pm 0.56 \mathrm{mV})$, indicating that the surface was modified with a carboxyl group. All the PDI $<0.3$ (Figure 4B), which meet the basic requirements for the effective size result, implied that the size distribution was narrow. 
A

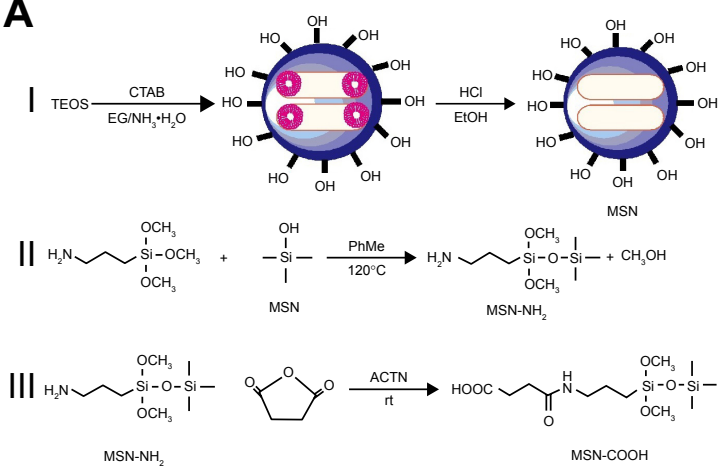

C

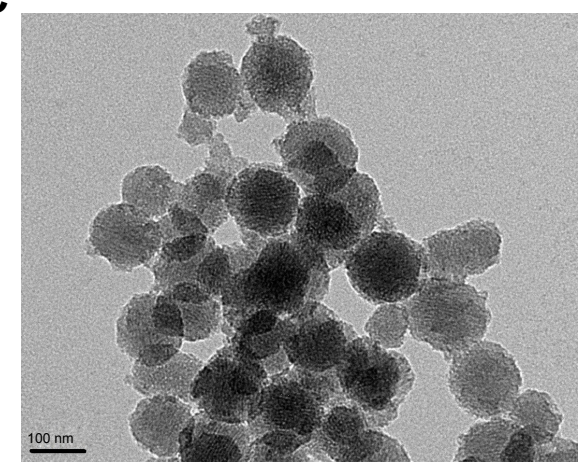

B

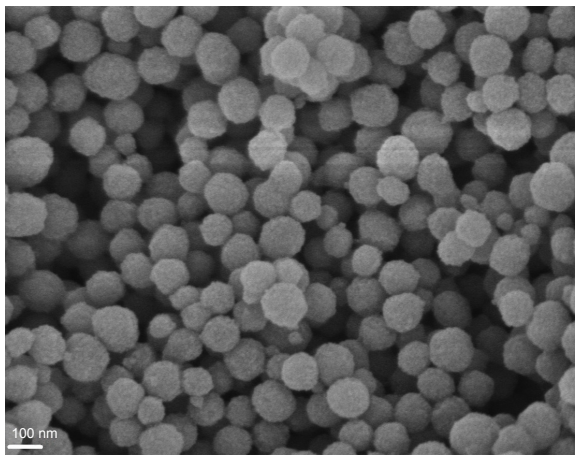

D

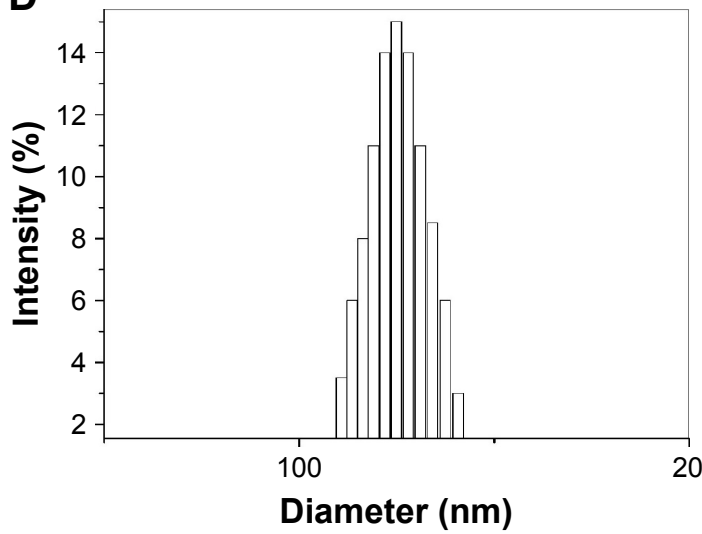

Figure 2 (A) Synthesis schemes of MSN (I), MSN-NH 2 (II), and MSN-COOH (III). (B) SEM images of MSN. (C) TEM images of MSN. (D) The size distribution of MSN determined by DLS (mean $\pm S D, n=5)$.

Abbreviations: ACTN, acetone; CTAB, cetyltrimethylammonium bromide; DLS, dynamic light scattering; EG, ethylene glycol; MSN, mesoporous silica nanoparticle; rt, room temperature; SEM, scanning electron microscopy; TEM, transmission electron microscopy; TEOS, tetraethyl orthosilicate.

Thus, the FT-IR spectra and zeta potentials revealed the successful incorporation of the amine and carboxyl groups.

TG analyzer was used to analyze TG profile of MSN, MSN/CTAB, MSN-NH , and MSN-COOH. The weight decrease in the TG curve of MSN/CTAB below $100^{\circ} \mathrm{C}$ was attributable to the loss of adsorbed water (Figure 4C). When heated to $300^{\circ} \mathrm{C}$, a significant weight loss was observed that was attributed to degradation of the surface-active agent CTAB. In the curve for MSN, the weight decrease from the loss of adsorbed water occurred below $100^{\circ} \mathrm{C}$. When the temperature was increased to $300^{\circ} \mathrm{C}$, the weight was $86.94 \%$ of the original value, indicating that the mesoporous structure of MSN was relatively stable, and that the CTAB was completely removed by repeated alcohol-acid reflux. ${ }^{41}$ The TG curves of amino- and carboxyl-modified MSN are shown in Figure 4C. Compared to the curve of MSN, a greater weight loss was observed for $\mathrm{MSN}-\mathrm{NH}_{2}$ and $\mathrm{MSN}-\mathrm{COOH}$ than for MSN after heating to $300^{\circ} \mathrm{C}$. This can be attributed to the
A

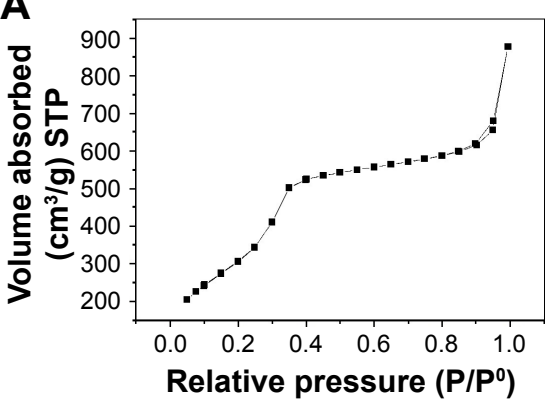

B

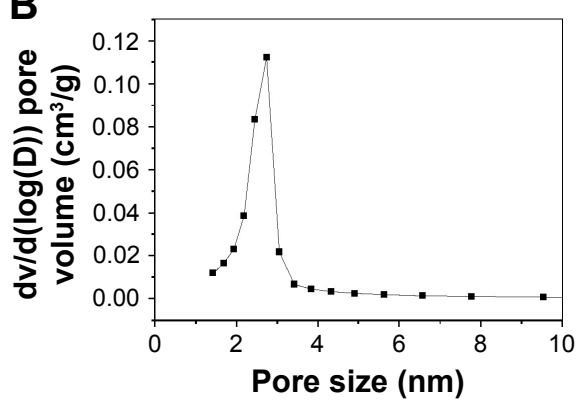

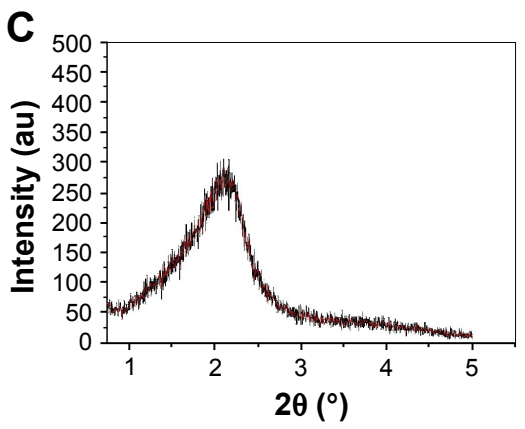

Figure 3 (A) Nitrogen adsorption-desorption isotherms, (B) pore size distribution, and (C) the small-angle XRD patterns of MSN. Abbreviations: MSN, mesoporous silica nanoparticle; XRD, X-ray diffraction. 

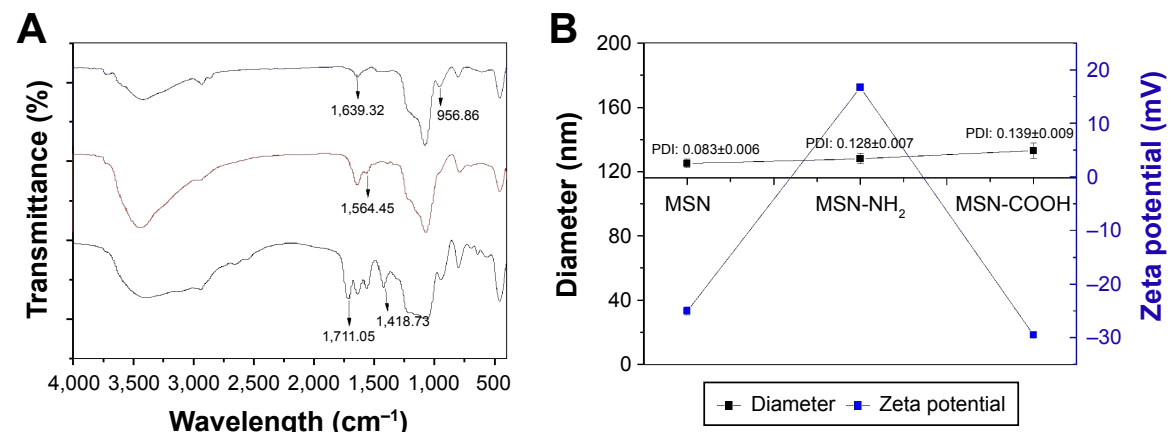

Wavelength $\left(\mathrm{cm}^{-1}\right)$

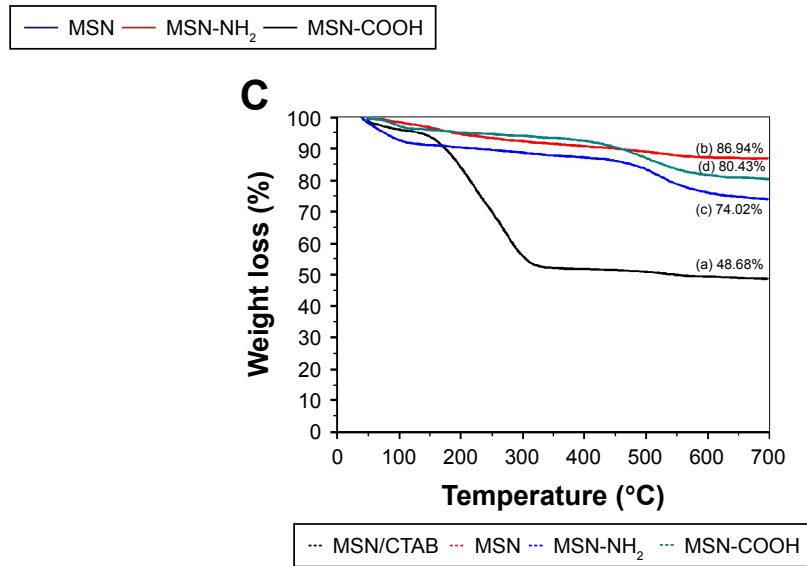

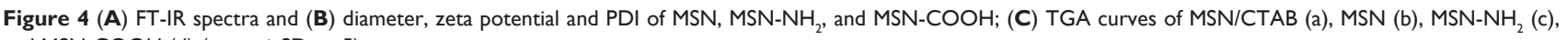
and $\mathrm{MSN}-\mathrm{COOH}$ (d) (mean $\pm \mathrm{SD}, \mathrm{n}=5$ ).

Abbreviations: CTAB, cetyltrimethylammonium bromide; FT-IR, Fourier transform infrared; MSN, mesoporous silica nanoparticle; PDI, polydispersity index; TGA, thermogravimetric analysis.

decomposition weights of the amino and carboxyl groups. These results confirmed that the MSNs were successfully functionalized.

The folate receptor is overexpressed in several types of human malignancy, including kidney, brain, lung, and breast cancers. Its ligand folate is a vitamin that is used for the biosynthesis of nucleotides and is present at high levels in tumors to meet growth requirements. To achieve tumor FA receptor targeting, we fabricated FA-grafted CS (FA-CS) as a membrane material according to the method outlined in Figure $5 \mathrm{~A}$ and characterized the structure by FT-IR (Figure 5B). The appearance of the peak at about 1,693 $\mathrm{cm}^{-1}$ was attributed to the ester linkage, whereas the signal at $1,655 \mathrm{~cm}^{-1}$ was ascribed to the aromatic ring of folate. These results confirm that folate moieties were conjugated to CS.

The functional membrane covering the MSN surface was prepared by the polyelectrolyte composite method or by LbL self-assembly. The polycation CS and polyanion HA polymers were used as raw materials. The presence of the membrane on MSNs was confirmed by measuring the zeta potential (Figure 6) and by TEM and DLS analyses (Figure 7). After coating the surface with CS or HA, the zeta potential of the nanoparticles changed alternately; values for
MSN-LbL (all layers), MSN-NH $-\mathrm{LbL}$ (all layers), and MSN-COOH-LbL (all layers) are shown in Figure 6A. The result indicated that a membrane made from polyelectrolyte material had already coated the surfaces of MSN, $\mathrm{MSN}_{-} \mathrm{NH}_{2}$, and MSN-COOH. The surface zeta potential $(\geq \pm 25 \mathrm{mV})$ reflects the stability of the colloid system; the higher zeta potential is associated with the higher stability of the colloid system; compared to LbL self-assembled on MSN and its derivatives $\mathrm{MSN}-\mathrm{NH}_{2}$ and $\mathrm{MSN}-\mathrm{COOH}$, the zeta potential of each polyelectrolyte layer on the $\mathrm{MSN}-\mathrm{NH}_{2}$ surface was larger and thus more stable. We examined the stability of the nanoparticles covered by membrane in aqueous medium by comparing their average size and appearance after storage for a given certain period. The native MSN showed obvious precipitation, and the MSN-COOH-LbL covered with a layer with low zeta potential also easily flocculated and precipitated. Therefore, membrane-controlled $\mathrm{MSN}-\mathrm{NH}_{2}$ samples were selected for physicochemical characterization and cell activity experiments.

We next examined the stability of $\mathrm{MSN}-\mathrm{NH}_{2}$ nanoparticles in different physiological solutions and found that the zeta potentials were larger in PBS and saline solution than in pure water (Figure 6B), implying that the particles were 
A

FA
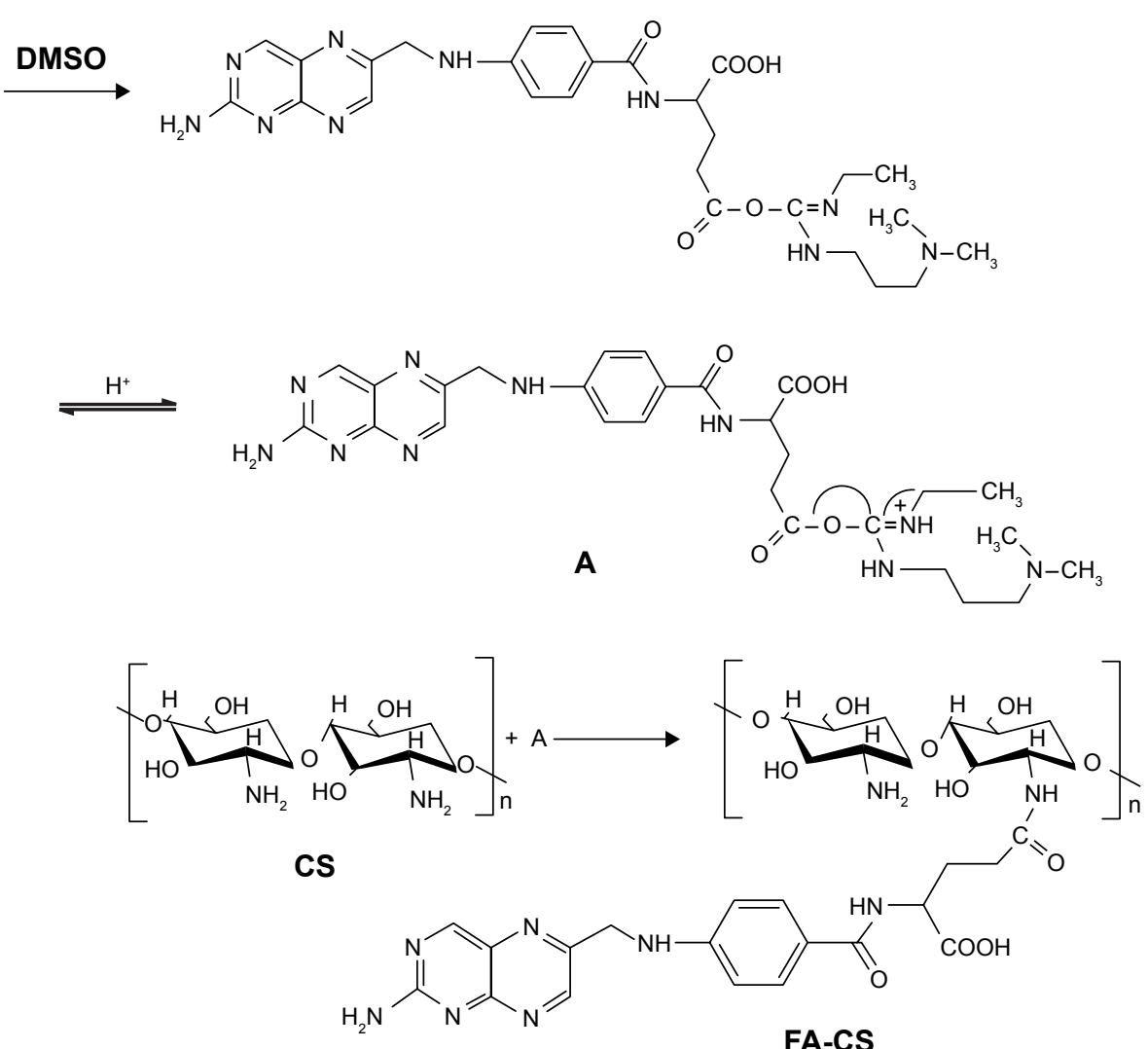

FA-CS

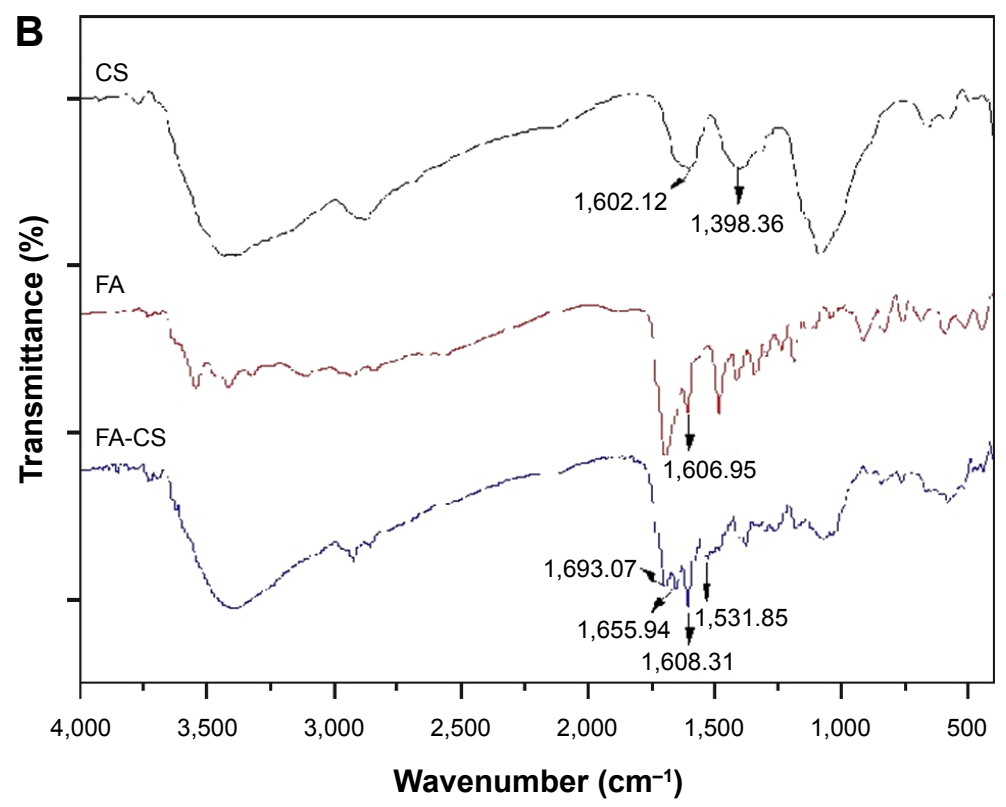

Figure 5 (A) Synthesis schemes of FA-CS. (B) FT-IR spectra of FA, CS, and FA-CS.

Abbreviations: CS, chitosan; DMSO, dimethyl sulfoxide; FA, folic acid; FA-CS, FA-modified CS; FT-IR, Fourier transform infrared. 


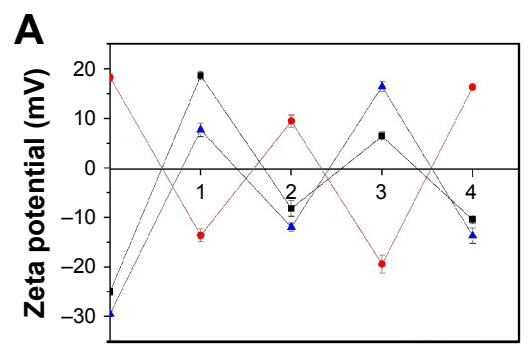

Layer number

$-\mathrm{MSN}-\mathrm{LbL} \rightarrow \mathrm{MSN}-\mathrm{NH}_{2}-\mathrm{LbL}$ $\rightarrow$ MSN-COOH-LbL

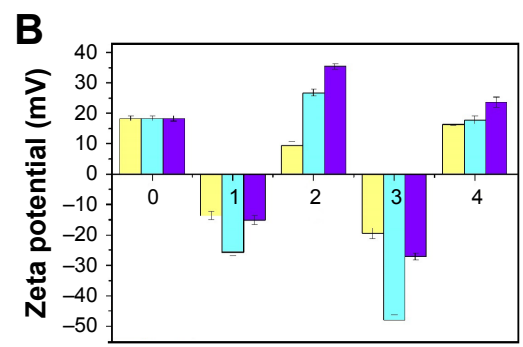

Layer number

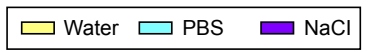

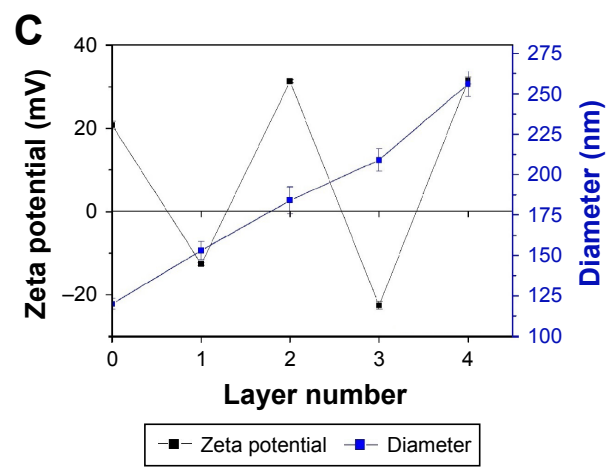

Figure 6 Zeta potential of $(\mathbf{A})$ LbL nanoparticles without DOX and (B) the MSN-NH 2 -LbL in different physiological conditions. (C) Zeta potential and diameter of DOX@ $\mathrm{MSN}-\mathrm{NH}_{2}(\mathrm{HA} / \mathrm{CS} / \mathrm{HA} / \mathrm{FA}-\mathrm{CS})$ (mean $\left.\pm \mathrm{SD}, \mathrm{n}=5\right)$.

Abbreviations: CS, chitosan; DOX, doxorubicin; FA, folic acid; FA-CS, FA-modified CS; HA, hyaluronic acid; LbL, layer-by-layer; MSN, mesoporous silica nanoparticle.

more stable under physiological conditions due to the effects of ions and that they have the potential to be used in the human body. In the same manner, DOX-loaded MSN-NH was also coated with HA and CS. To increase the targeting of membrane-decorated nanoparticles, FA-fabricated CS was used to cover the nanoparticle surface. After the addition of four layers (labeled DOX@MSN-NH 2 [HA/CS/HA/ FA-CS]), the size was about $256 \mathrm{~nm}(\mathrm{PDI}=0.182 \pm 0.007)$ and the nanoparticle was stable in medium with a zeta potential of about $+31 \mathrm{mV}$ (Figure 6C).

A $\mathrm{CD}_{44}$ receptor-targeting membrane-controlled nanoparticle with three layers (with the outer face decorated by HA) was also prepared (labeled DOX@MSN-NH ${ }_{2}[\mathrm{HA} / \mathrm{CS} /$ HA]). MSN-NH $\mathrm{N}_{2}$ nanoparticles were covered with smooth layers of polyelectrolyte membrane (Figure 7A). The morphology of the composite nanoparticles suggested a functional membrane coating the $\mathrm{MSN}-\mathrm{NH}_{2}$ surface, with a diameter determined by DLS of $209 \mathrm{~nm}$ (PDI $=0.167 \pm 0.009$; Figure 7B).
The number of layers to be used in the final formulation was determined based on both the size and the zeta potential. Nanoparticles with 1 or 2 layers exhibited a lower zeta potential and easily flocculated and precipitated due to the weak membrane on the surface. When the number of nanoparticle layers increased to 5 or more, the size of the nanoparticles also increased to greater than $300 \mathrm{~nm}$, which was not suitable for targeted drug delivery systems administrated by a systemic route. Hence, samples with three and four layers were selected in the final formulation.

\section{In vitro drug release}

The capacity of composite nanoparticles for $\mathrm{pH}$-triggered payload release was characterized using DOX as a model drug. Since the characteristic absorption peak of DOX is around $483 \mathrm{~nm}$, DOX-loading and entrapment efficiency of nanoparticles was determined by UV-Vis spectroscopy and calculated using a standard curve equation (Figure S1). ${ }^{42}$ The DL efficiency of MSN, MSN-NH $\mathrm{N}_{2}, \mathrm{MSN}-\mathrm{COOH}$ and
A

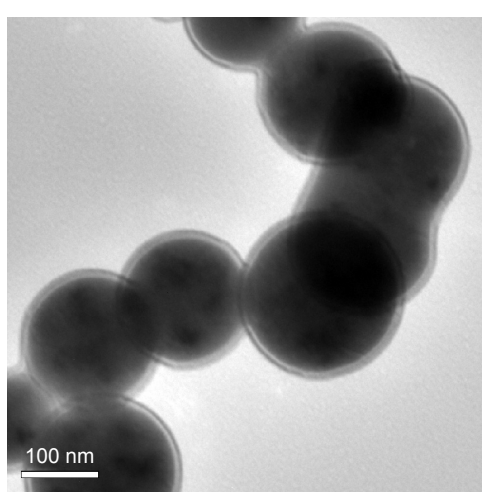

B

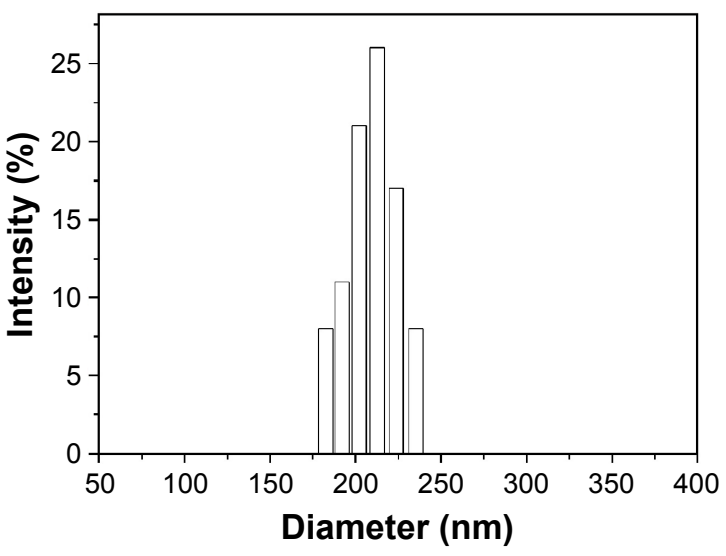

Figure 7 TEM images (A) and size distribution (B) of DOX@MSN-NH $\mathrm{H}_{2}(\mathrm{HA} / \mathrm{CS} / \mathrm{HA})($ mean $\pm \mathrm{SD}, \mathrm{n}=5)$.

Abbreviations: CS, chitosan; DOX, doxorubicin; HA, hyaluronic acid; MSN, mesoporous silica nanoparticle; TEM, transmission electron microscopy. 
DOX@MSN-NH ${ }_{2}$ (HA/CS/HA/FA-CS) was 23.27\% $\pm 0.27 \%$, $18.26 \% \pm 1.24 \%, 25.29 \% \pm 0.23 \%$, and $28.71 \% \pm 0.39 \%$, respectively, while entrapment efficiency was $53.62 \% \pm 1.23 \%$, $42.24 \% \pm 2.17 \%, 54.12 \% \pm 1.35 \%$, and $59.31 \% \pm 1.43 \%$, respectively. Compared with the loading efficiency of other nanoparticles, the highest loading efficiency of the nanoparticles after LbL coating is probably that during the drug loading: the washing was performed after the membrane coating process for the LbL coating samples and then the membrane on the surface allowed the drug to be effectively encapsulated in the nanoparticle protected against drug leakage.

To study the $\mathrm{pH}$-dependent drug release characteristics, two different media with $\mathrm{pH}=7.4$ and $\mathrm{pH}=5.0$ were selected to simulate the environment of blood and the tumor region, respectively. The release performance of the four different nanoparticles under these conditions was evaluated over $48 \mathrm{~h}$ at $37^{\circ} \mathrm{C}$.

The cumulative drug release ratios of DOX@MSN, DOX@MSN-NH, DOX@MSN-COOH, and the membranemodified DOX@MSN-NH 2 (HA/CS/HA/FA-CS) at pH 7.4 were $52.65 \% \pm 1.45 \%, 33.22 \% \pm 3.90 \%, 37.06 \% \pm 0.63 \%$, and $25.40 \% \pm 2.24 \%$, respectively (Figure $8 \mathrm{~A}$ ). At pH 5.0, the ratios were $84.29 \% \pm 4.08 \%, 47.36 \% \pm 4.44 \%, 43.53 \% \pm 1.89 \%$, and $69.14 \% \pm 1.89 \%(n=3)$, respectively (Figure $8 B$ ). Thus, these four types of nanoparticles are more likely to release loaded DOX under acidic conditions, such as that in the tumor microenvironment. Once the drug-loaded nanoparticles are taken up by tumor cells, they quickly release the drug, thereby specifically targeting the tumor. Under physiological conditions, DOX is released slowly from nanoparticles that persist in the body for a long time; this can reduce the toxic side effects of DOX on normal tissues. Compared to unmodified DOX@MSN,DOX@MSN-NH MSN-COOH, DOX@MSN-NH 2 (HA/CS/HA/FA-CS) coated with membrane exhibited higher $\mathrm{pH}$ sensitivity and, accordingly, released less under physiological condition than under acidic conditions. Thus, membrane coating of nanoparticles can reduce side effects on normal tissue and prolong the circulation time of drug carriers to maintain an adequate concentration of the drug in the blood.

\section{In vitro cellular uptake}

DOX exhibits red fluorescence at $488 \mathrm{~nm}$. After staining nuclei with Hoechst 33258, we obtained images of cells at two different excitation wavelengths; the overlay of the two images revealed whether the nanoparticles were taken up by cells (Figure 9). Free DOX was internalized by HepG2 cells and distributed in or around the nucleus (Figure 9A). Compared to the Figure 9B and C, the fluorescence intensity of the membrane-controlled nanoparticles was comparatively higher than that of the nanoparticle without membrane, and the drug was mainly detected in the endosomes and lysosomes (Figure 9D and E). By using blue-fluorescent Hoechst 33258 as an indicator, after $2 \mathrm{~h}$ of cultivation, red fluorescence of DOX could be observed in both the cytosol and nucleus, indicating a release of DOX from the membrane decorated nanoparticles. The results further indicate that the membrane-decorated nanoparticles are an effective intracellular drug delivery system that could target the cancer region by specific recognition of the FA
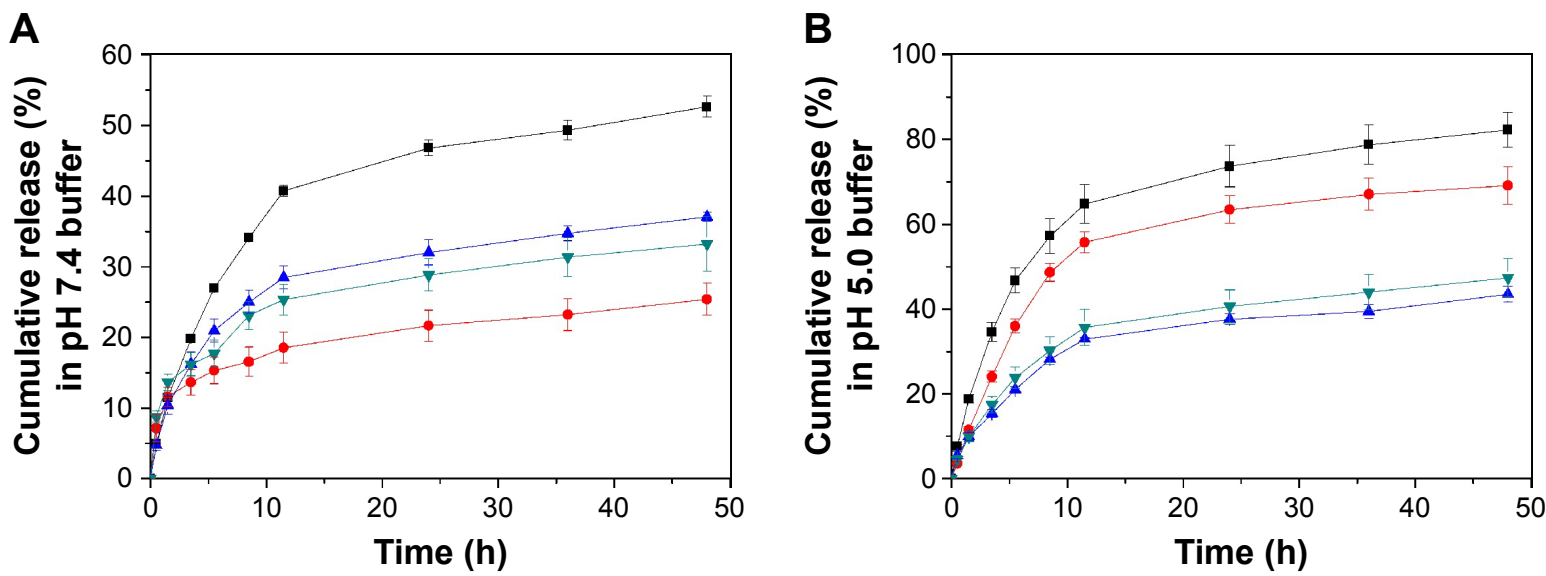

$\rightarrow$ DOX@MSN $\rightarrow$ DOX@MSN-COOH $\rightarrow$ DOX@MSN-NH ${ }_{2} \rightarrow$ DOX@MSN-NH ${ }_{2}(\mathrm{HA} / \mathrm{CS} / \mathrm{HA} / \mathrm{FA}-\mathrm{CS})$

Figure 8 In vitro release profiles from DOX@MSN, DOX@MSN/NH ${ }_{2}$, DOX@MSN-COOH, and DOX@MSN-NH $2(\mathrm{HA} / \mathrm{CS} / \mathrm{HA} / \mathrm{FA}-\mathrm{CS})$ core-shell particles at 37 ${ }^{\circ} \mathrm{C}$ in $\mathrm{pH} 7.4$ buffer $(\mathbf{A})$ and in $\mathrm{pH} 5.0$ buffer (B).

Notes: Data are represented as mean \pm SD $(n=3)$.

Abbreviations: CS, chitosan; DOX, doxorubicin; FA, folic acid; FA-CS, FA-modified CS; HA, hyaluronic acid; MSN, mesoporous silica nanoparticle. 

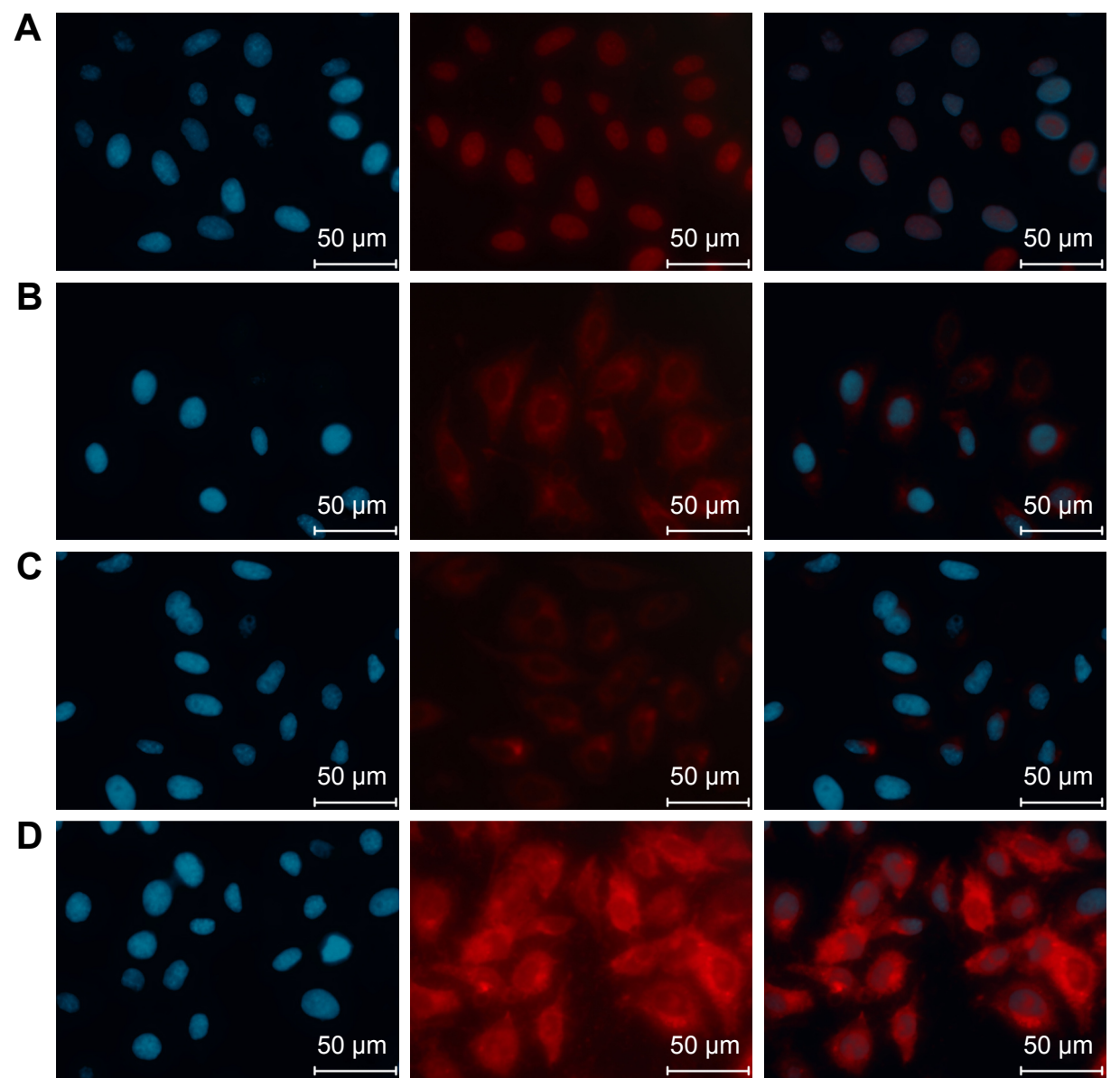

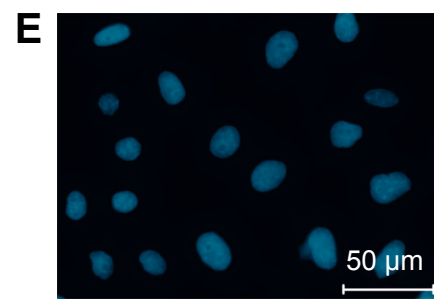

Hoechst 33,258

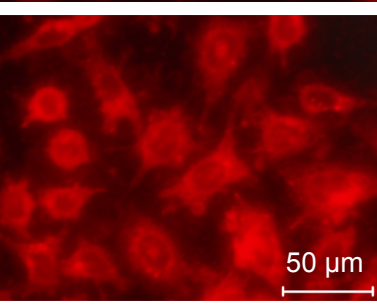

DOX

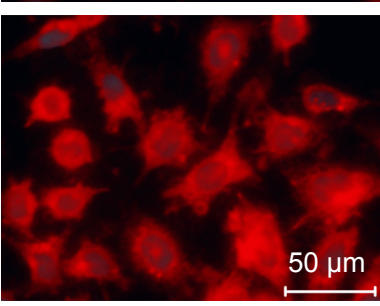

Merge

Figure 9 Representative fluorescence microscopy of HepG2 with (A) DOX, (B) DOX@MSN-NH ${ }_{2}$, (C) DOX@MSN-COOH, (D) DOX@MSN-NH ${ }_{2}(\mathrm{HA} / C S / H A)$, and (E) DOX@MSN-NH $\mathrm{NH}_{2}(\mathrm{HA} / \mathrm{CS} / \mathrm{HA} / \mathrm{CS}-\mathrm{FA})$ after administration for $2 \mathrm{~h}$.

Abbreviations: CS, chitosan; DOX, doxorubicin; FA, folic acid; FA-CS, FA-modified CS; HA, hyaluronic acid; MSN, mesoporous silica nanoparticle.

receptor or $\mathrm{CD}_{44}$ receptors on the tumor cell surface, hold DOX until endocytosis, and then release the drug within the lysosomes by the acid-triggered dissolution of the coating membrane. ${ }^{43}$

To improve the drug delivery efficiency, we designed DOX@MSN-NH ${ }_{2}(\mathrm{HA} / \mathrm{CS} / \mathrm{HA} / \mathrm{FA}-\mathrm{CS})$ and DOX@ $\mathrm{MSN}-\mathrm{NH}_{2}(\mathrm{HA} / \mathrm{CS} / \mathrm{HA})$ to further evaluate the receptortargeted release properties of the membrane-controlled nanoparticles, and the competitive inhibitory effects of FA and HA on cellular uptake were investigated by CLSM. The folate receptor or $\mathrm{CD}_{44}$ receptor was blocked on the surface of HepG2 cells by pre-cultivating HepG2 cells with free folate or
HA for $2 \mathrm{~h} \cdot{ }^{39}$ As shown in Figure 10, after incubation for $2 \mathrm{~h}$, the nanoparticles in two different culture media were taken up by HepG2 cells. However, the fluorescence intensity of nanoparticles was lower in medium with FA and HA compared to in medium without FA and HA. This suggests that nanoparticles coated with FA or HA, which allows targeting to the $\mathrm{FA}$ or $\mathrm{CD}_{44}$ receptors on the HepG2 cell surface, were inhibited by free FA and HA molecules in the medium to some extent. In contrast, nanoparticles in the medium lacking FA and HA were readily taken up by the cells. Thus, the results clearly indicate that membrane-controlled DOX@ MSN-NH ${ }_{2}(\mathrm{HA} / \mathrm{CS} / \mathrm{HA} / \mathrm{FA}-\mathrm{CS})$ and DOX@MSN-NH${ }_{2}(\mathrm{HA} /$ 


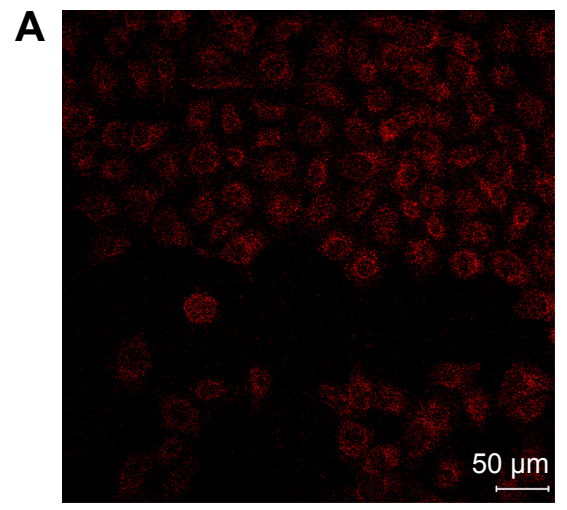

HA (-)

DOX@MSN-NH

(HA/CS/HA)

B

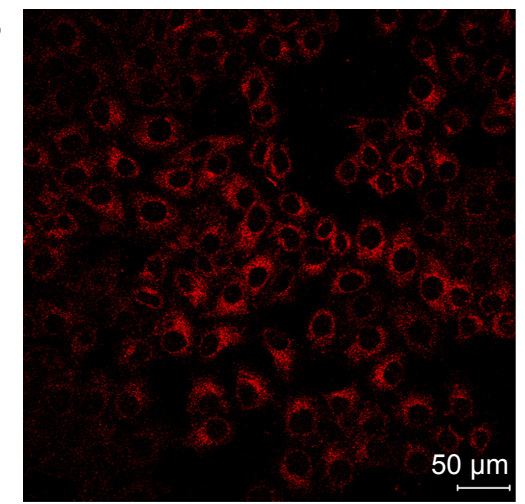

FA $(-)$

DOX@MSN-NH ${ }_{2} \quad(+)$

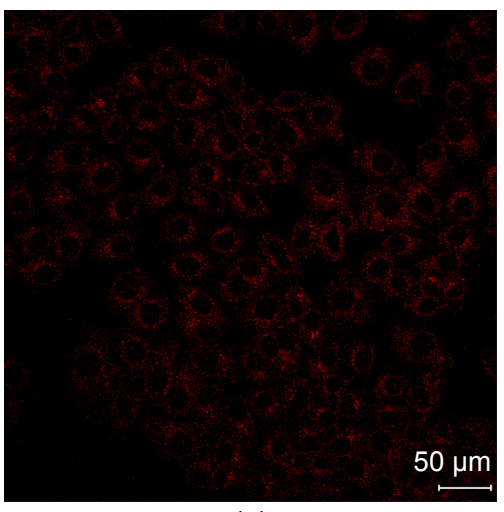

$(+)$

(+)

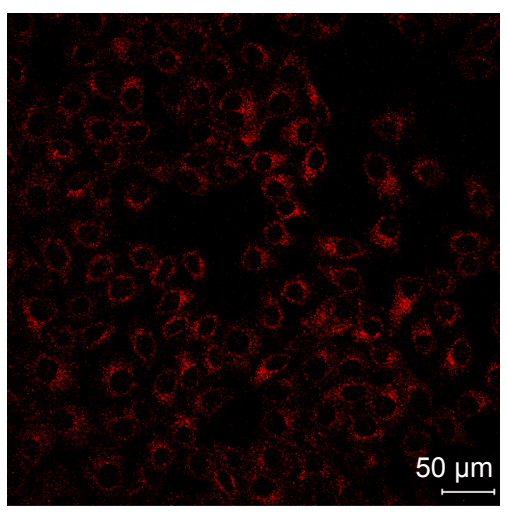

$(+)$

$(+)$

Figure 10 CLSM images of HepG2 cells after incubation with (A) DOX@MSN-NH $(\mathrm{HA} / \mathrm{CS} / \mathrm{HA})$ in the culture medium without HA or containing HA for 2 h and (B) DOX@MSN-NH $\mathrm{H}_{2}(\mathrm{HA} / \mathrm{CS} / \mathrm{HA} / \mathrm{FA}-\mathrm{CS})$ in the culture medium without FA or containing FA for $2 \mathrm{~h}$.

Abbreviations: CLSM, confocal laser scanning microscopy; CS, chitosan; DOX, doxorubicin; FA, folic acid; FA-CS, FA-modified CS; HA, hyaluronic acid; MSN, mesoporous silica nanoparticle.

CS/HA) have tumor cell-targeting capacity and were an effective delivery system for targeted anticancer drugs.

\section{Cytotoxicity of membrane-controlled MSN}

The biocompatibility of bare and DOX-loaded MSNs was evaluated in L02 and HepG2 cells with the MTT assay. After $24 \mathrm{~h}$ of incubation, the blank nanoparticles showed low toxicity toward L02 cells, and the cell survival remained $>80 \%$ at concentrations higher than $20 \mu \mathrm{g} / \mathrm{mL}$ (Figure 11A). According to the relationship between cell proliferation rate (survival) and degree of cytotoxicity, ${ }^{44}$ since the concentration was $<20 \mu \mathrm{g} / \mathrm{mL}$ and cell survival rate was $>80 \%$, samples in the concentration range $<20 \mu \mathrm{g} / \mathrm{mL}$ were not toxic to cells.

We evaluated the cytotoxicity of different concentrations of free DOX,DOX@MSN,DOX@MSN-NH $\mathrm{COOH}$, and DOX@MSN-NH $2(\mathrm{HA} / \mathrm{CS} / \mathrm{HA})$ to HepG2 and
L02 cells after $24 \mathrm{~h}$ of incubation. Compared to HepG 2 cells (Figure 11B), L02 cells showed higher viability in the presence of DOX@MSN-NH $2(\mathrm{HA} / \mathrm{CS} / \mathrm{HA})$ at every concentration tested (Figure 11C), indicating that the former cells were more sensitive to membrane-controlled MSN. We calculated the half-maximal inhibitory concentration $\left(\mathrm{IC}_{50}\right)$ of drugloaded nanoparticles in HepG2 cells and L02 cells based on the percentage of cells surviving at different concentrations (Figure 11D). The $\mathrm{IC}_{50}$ of free DOX and DOX@MSN$\mathrm{NH}_{2}(\mathrm{HA} / \mathrm{CS} / \mathrm{HA})$ in HepG2 cells was 0.53 and $0.45 \mu \mathrm{g} / \mathrm{mL}$, respectively, indicating that the latter is more toxic. In L02 cells, membrane-controlled MSNs were less toxic, with an $\mathrm{IC}_{50}$ of 1.57, 5.37, 6.34, 6.83, and 8.38 for DOX, DOX@ MSN,DOX@MSN-NH,DOX@MSN-COOH, and DOX@ MSN-NH 2 (HA/CS/HA), respectively. This indicates that the $\mathrm{pH}$ sensitivity and high cellular uptake by receptor targeting enhanced the antitumor effect of the composite nanoparticles and can reduce side effects to normal cells. 

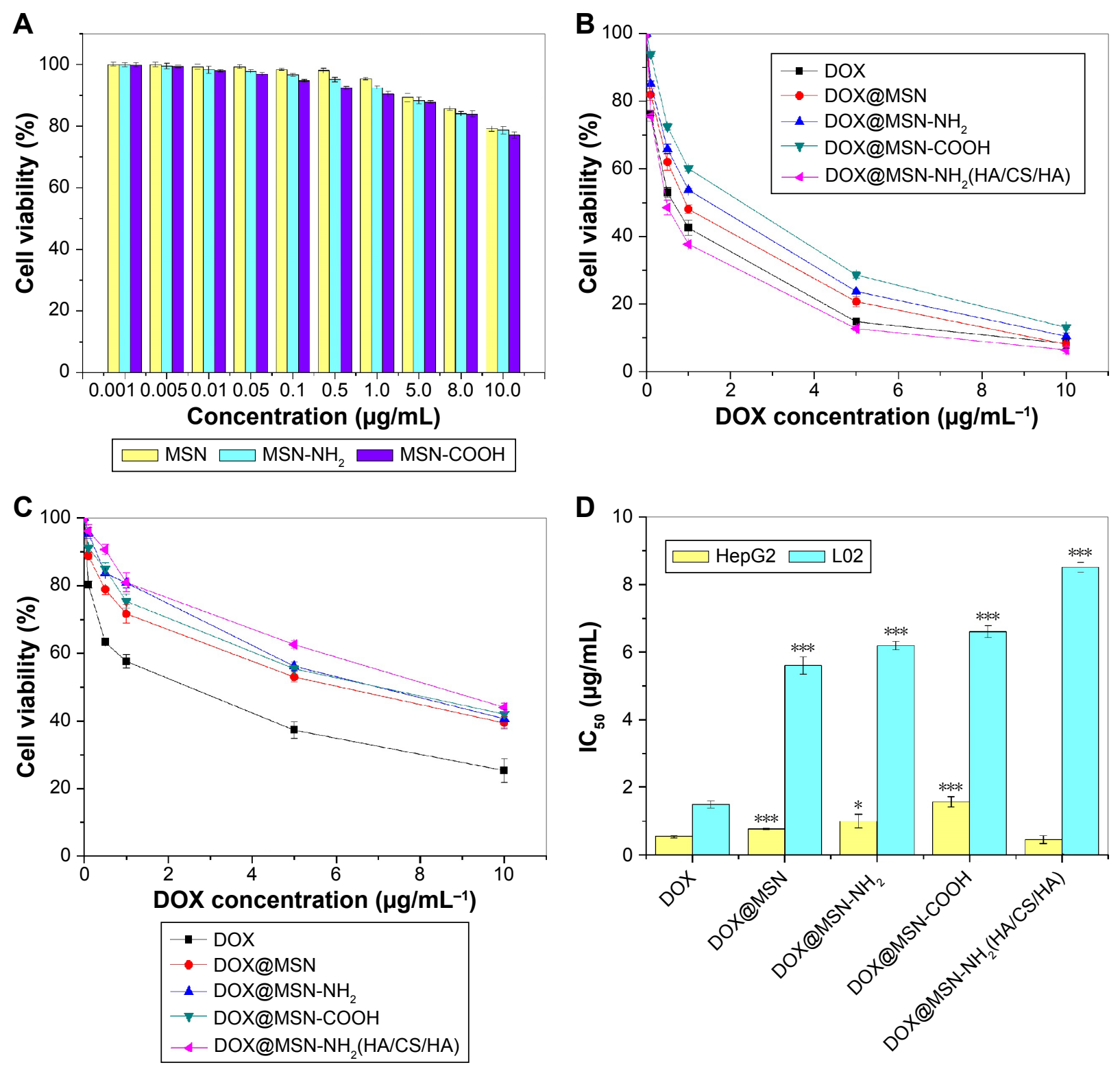

Figure II In vitro cytotoxicity assay curves of blank MSN and modified MSN carrier (A) on L02 cells. In vitro cytotoxicity assay curves of DOX, DOX@MSN, DOX@ MSN-NH ${ }_{2}$, DOX@MSN-COOH, DOX@MSN-NH ${ }_{2}(\mathrm{HA} / \mathrm{CS} / \mathrm{HA})$ on HepG2 (B) and L02 cells (C). (D) The IC ${ }_{50}$ value to HepG2 cells and L02 cells. Data are represented as mean \pm SD $(n=5)$. Statistical analysis versus the DOX group: ${ }^{*} p<0.05 ; * * *<0.00$ I.

Abbreviations: CS, chitosan; DOX, doxorubicin; HA, hyaluronic acid; $\mathrm{IC}_{50}$, the half-maximal inhibitory concentration; MSN, mesoporous silica nanoparticle.

It is well known that in HepG2 cells or other types of cancer cells, overexpression of folate receptors and $\mathrm{CD}_{44}$ receptors occurs. Nanoparticles with modification of FA or HA were easily targeted to the cancer region by specific recognition of the FA receptor or $\mathrm{CD}_{44}$ receptors on the tumor cell surface. Once the nanoparticles were taken up by the cancer cells, the DOX in the membrane-decorated nanoparticles was intracellularly released from the acid-sensitive membrane in the specific acid tumor cell environment. On the one hand, the membrane was helpful for reducing the drug leakage and enhancing the stability of the nanoparticles in the transmission process. On the other hand, the membrane was a specific switch, which had the ability to recognize the cancer cells and stimulus response to open the warehouse. This type of multifunctional integration of targeting and stimulus response might give rise to specific cytotoxicity toward cancer cells but not normal cells. Moreover, as seen in the cytotoxicity assay, either HA or FA can be selected as the targeting ligand.

\section{Conclusion}

The novel membrane-controlled nanoparticle delivery system developed in this study combined the high DL capacity of MSN and the biocompatibility and capacity for $\mathrm{pH}$ responsive controlled release of natural membrane materials. 
The tumor cell surface receptor targeting by the nanoparticles can improve treatment efficacy while reducing side effects to normal cells. Hence, this system has great potential to be employed for the targeted cancer treatment. Furthermore, based on the membrane-controlled drug delivery system, fluorescent quantum dots can also be loaded onto the MSNs and combined with tracer imaging to allow simultaneous tumor imaging and therapy to evaluate the targeting behavior of the nanocomposite. ${ }^{45}$

\section{Acknowledgment}

The authors are grateful for the financial support from the National Natural Science Foundation of China (81401510), Hubei Provincial Natural Science Foundation of China (2017CFB414), the Academic Team of SouthCentral University for Nationalities (CZW15017), the Fundamental Research Funds for the Central Universities, South-Central University for Nationalities (CZP17092), and the National College Students Innovation and Entrepreneurship Training Project (GCX16034).

\section{Disclosure}

The authors report no conflicts of interest in this work.

\section{References}

1. Mignani S, Bryszewska M, Klajnert-Maculewicz B, Zablocka M, Majoral JP. Advances in combination therapies based on nanoparticles for efficacious cancer treatment: an analytical report. Biomacromolecules. 2015;16(1):1-27.

2. Ma L, Kohli M, Smith A. Nanoparticles for combination drug therapy. ACS Nano. 2013;7(11):9518-9525.

3. Mandal B, Bhattacharjee H, Mittal N, et al. Core-shell-type lipid-polymer hybrid nanoparticles as a drug delivery platform. Nanomedicine. 2013;9(4):474-491.

4. Teo PY, Cheng W, Hedrick JL, Yang YY. Co-delivery of drugs and plasmid DNA for cancer therapy. Adv Drug Deliv Rev. 2016;98:41-63.

5. Zhang Y, Chan HF, Leong KW. Advanced materials and processing for drug delivery: the past and the future. Adv Drug Deliv Rev. 2013; 65(1):104-120.

6. Ashley CE, Carnes EC, Phillips GK, et al. The targeted delivery of multicomponent cargos to cancer cells via nanoporous particle-supported lipid bilayers. Nat Mater. 2011;10:389-397.

7. Chen Y, Chen H, Zhang S, et al. Multifunctional mesoporous nanoellipsoids for biological bimodal imaging and magnetically targeted delivery of anticancer drugs. Adv Funct Mater. 2011;21:270-279.

8. Yao XX, Tian ZF, Liu JX, Zhu YF, Hanagata N. Mesoporous silica nanoparticles capped with graphene quantum dots for potential chemo-photothermal synergistic cancer therapy. Langmuir. 2017;33(2): 591-599.

9. Torney F, Trewyn BG, Lin VS-Y, Wang K. Mesoporous silica nanoparticles deliver DNA and chemicals into plants. Nat Nanotechnol. 2007; 2(5):295-300.

10. Li YH, Li N, Pan W, Yu ZZ, Yang LM, Tang B. Hollow mesoporous silica nanoparticles with tunable structures for controlled drug delivery. ACS Appl Mater Interfaces. 2017;9(3):2123-2129.

11. Heleg-Shabtai V, Aizen R, Sharon E, et al. Gossypol-capped mitoxantrone-loaded mesoporous $\mathrm{SiO} 2 \mathrm{NPs}$ for the cooperative controlled release of two anti-cancer drugs. ACS Appl Mater Interfaces. 2016;8(23):14414-14422.
12. Chen TC, Wu W, Xiao H, Chen YX, Chen M, Li JS. Intelligent drug delivery system based on mesoporous silica nanoparticles coated with an ultra-pH-sensitive gatekeeper and poly(ethylene glycol). ACS Macro Lett. 2016;5(1):55-58.

13. Allen T M, Cullis PR. Liposomal drug delivery systems: from concept to clinical applications. Adv Drug Deliv Rev. 2013;65(1):36-48.

14. Deng JZ, Morton WS, Ben-Akiva E, Dreaden CE, Shopsowitz EK, Paula T. Hammond layer-by-layer nanoparticles for systemic codelivery of an anticancer drug and siRNA for potential triple-negative breast cancer treatment. ACS Nano. 2013;7:9571-9584.

15. Lee MH, Kim EJ, Lee H, et al. Liposomal texaphyrin theranostics for metastatic liver cancer. $J$ Am Chem Soc. 2016;138(50):16380-16387.

16. Das M, Singh RP, Datir SR, Jain S. Surface chemistry dependent "switch" regulates the trafficking and therapeutic performance of drugloaded carbon nanotubes. Bioconjug Chem. 2013;24:626-639.

17. Mieszawska AJ, Kim Y, Gianella A, et al. Synthesis of polymer-lipid nanoparticles for image-guided delivery of dual modality therapy. Bioconjug Chem. 2013;24(9):1429-1434.

18. Rizzo LY, Theek B, Storm G, Kiessling F, Lammers T. Recent progress in nanomedicine: therapeutic, diagnostic and theranostic applications. Curr Opin Biotechnol. 2013;24(6):1159-1166.

19. Maeda H, Wu J, Sawa T, Matsumura Y, Hori K. Tumor vascular permeability and the EPR effect in macromolecular therapeutics: a review. J Control Release. 2000;65(1-2):271-282.

20. Wu J, Sailor MJ. Chitosan hydrogel-capped porous $\mathrm{SiO} 2$ as a $\mathrm{pH}$ responsive nano-valve for triggered release of insulin. Adv Funct Mater. 2009;19:733-741.

21. Surace C, Arpicco S, Dufay-Wojcicki A, et al. Lipoplexes targeting the $\mathrm{CD}_{44}$ hyaluronic acid receptor for efficient transfection of breast cancer cells. Mol Pharm. 2009;6(4):1062-1073.

22. Yang C, Cao M, Liu H, et al. The high and low molecular weight forms of hyaluronan have distinct effects on $\mathrm{CD}_{44}$ clustering. $J$ Biol Chem. 2012;287(51):43094-43107.

23. Wang Z, Tian Y, Zhang H, et al. Using hyaluronic acid-functionalized $\mathrm{pH}$ stimuli responsive mesoporous silica nanoparticles for targeted delivery to $\mathrm{CD}_{44}$-overexpressing cancer cells. Int J Nanomed. 2016;11: 6485-6497.

24. Bitar A, Ahmad NM, Fessi H, Elaissari A. Silica-based nanoparticles for biomedical applications. Drug Discov Today. 2012;17(19-20): $1147-1154$

25. Castillo RR, Colilla M, Vallet-Regí M. Advances in mesoporous silicabased nanocarriers for co-delivery and combination therapy against cancer. Expert Opin Drug Deliv. 2017;14(2):229-243.

26. Kresge CT, Leonowicz ME, Roth WJ, Vartuli JC, Beck JS. Ordered mesoporous molecular-sieves synthesized by a liquid-crystal template mechanism. Nature. 1992;359:710-712.

27. Lai CY, Trewyn BG, Jeftinija DM, et al. A mesoporous silica nanosphere-based carrier system with chemically removable CdS caps for stimuli-responsive controlled release of neurotransmitters and drug molecules. J Am Chem Soc. 2003;125:4451-4459.

28. Zhu J, Niu Y, Li Y, et al. Stimuli-responsive delivery vehicles based on mesoporous silica nanoparticles: recent advances and challenges. J Mater Chem B. 2017;5(7):1339-1352.

29. Mamaeva V, Sahlgren C, Lindén M. Mesoporous silica nanoparticles in medicine-Recent advances. Adv Drug Deliv Rev. 2013;65: 689-702.

30. He Q, Zhang J, Shi J, et al. The effect of PEGylation of mesoporous silica nanoparticles on nonspecific binding of serum proteins and cellular responses. Biomaterials. 2010;31(6):1085-1092.

31. Chang B, Chen D, Wang Y, et al. Bioresponsive controlled drug release based on mesoporous silica nanoparticles coated with reductively sheddable polymer shell. Chem Mater. 2013;25:574-585.

32. Chang B, Guo J, Liu C, Qian J, Yang W. Surface functionalization of magnetic mesoporous silica nanoparticles for controlled drug release. J Mater Chem. 2010;20:9941-9947.

33. Luo Z, Hu Y, Xin R, et al. Surface functionalized mesoporous silica nanoparticles with natural proteins for reduced immunotoxicity. J Biomed Mater Res A. 2014;102(11):3781-3794. 
34. Qu D, Lin H, Zhang N, Xue J, Zhang C. In vitro evaluation on novel modified chitosan for targeted antitumor drug delivery. Carbohydr Polym. 2013;92:545-554.

35. Zou Z, He X, He D, et al. Programmed packaging of mesoporous silica nanocarriers for matrix metalloprotease 2-triggered tumor targeting and release. Biomaterials. 2015;58:35-45.

36. Chai F, Sun L, He X, et al. Doxorubicin-loaded poly (lactic-coglycolic acid) nanoparticles coated with chitosan/alginate by layer by layer technology for antitumor application. Int J Nanomed. 2017;12: 1791-1802.

37. Zhao W, Jiang X, Ni S, et al. Layer-by-layer self-assembly of polyelectrolyte multilayers on silica spheres as reversed-phase/hydrophilic interaction mixed-mode stationary phases for high performance liquid chromatography. J Chromatogr A. 2017;1499:111-117.

38. Poon Z, Lee JB, Morton SW, Hammond PT. Controlling in vivo stability and biodistribution in electrostatically assembled nanoparticles for systemic delivery. Nano Lett. 2011;11(5):2096-2103.

39. Xie M, Shi H, Lia Z, et al. A multifunctional mesoporous silica nanocomposite for targeted delivery, controlled release of doxorubicin and bioimaging. Colloids Surf B Biointerfaces. 2013;110:138-147.
40. Hu Y, Dong X, Ke L, et al. Polysaccharides/mesoporous silica nanoparticles hybrid composite hydrogel beads for sustained drug delivery. J Mater Sci. 2016;52:3095-3109.

41. Hu X, Wang Y, Peng B. Chitosan-capped mesoporous silica nanoparticles as $\mathrm{pH}$-responsive nanocarriers for controlled drug release. Chem Asian J. 2014;9(1):319-327.

42. Bibby DC, Taknadge JE, Dalal MK, et al. Pharmacokinetics and biodistribution of RGD-targeted doxorubicin-loaded nanoparticles in tumor-bearing mice. Int J Pharm. 2005;293(1/2):281-290.

43. Shi H, He X, Yuan Y, Wang K, Liu D. Nanoparticle-based biocompatible and long-life marker for lysosome labeling and tracking. Anal Chem. 2010;82(6):2213-2220.

44. Lavasanifar A, Samuel J, Kwon GS. Poly(ethylene oxide)-blockpoly(1-amino acid) micelles for drug delivery. Adv Drug Deliv Rev. 2002;54(2):169-190.

45. Choi KY, Liu G, Lee S, Chen X. Theranostic nanoplatforms for simultaneous cancer imaging and therapy: current approaches and future perspectives. Nanoscale. 2012;4(2):330-342. 


\section{Supplementary materials}

Table SI Physicochemical properties of MSN, MSN-NH ${ }_{2}$, and MSN-COOH (mean \pm SD, $n=5$ )

\begin{tabular}{llll}
\hline Sample & Size $(\mathbf{n m})$ & PDI & Zeta potential (mV) \\
\hline MSN & $125 \pm 2.6$ & $0.083 \pm 0.006$ & $-25.03 \pm 0.75$ \\
$M S N-N H_{2}$ & $128 \pm 3.1$ & $0.128 \pm 0.007$ & $16.73 \pm 0.42$ \\
MSN-COOH & $133 \pm 4.8$ & $0.139 \pm 0.009$ & $-29.5 \pm \pm 0.56$ \\
\hline
\end{tabular}

Abbreviations: MSN, mesoporous silica nanoparticle; PDI, polydispersity index.

A

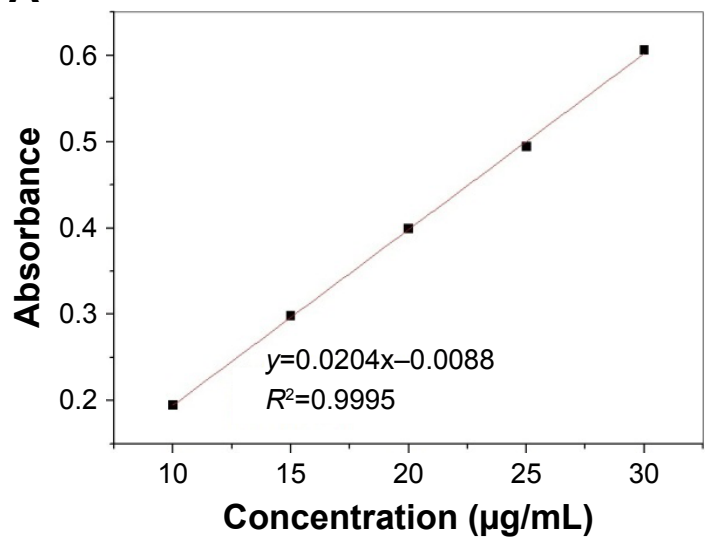

B

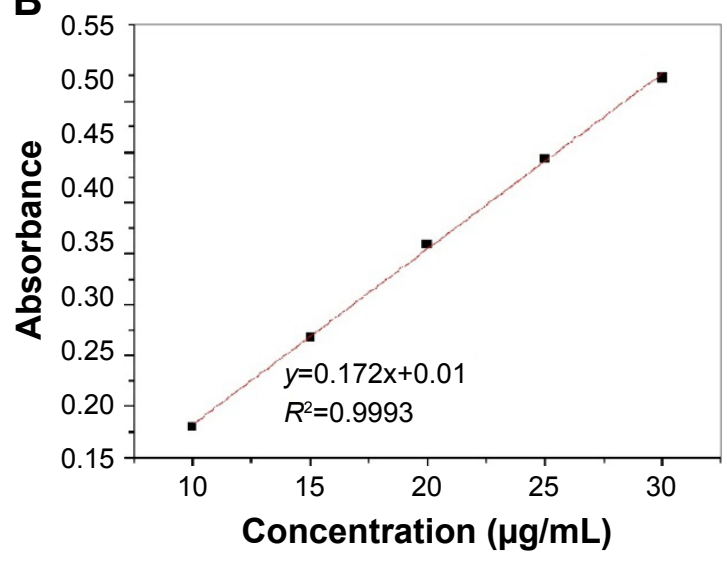

Figure SI Criterion curve of DOX at $(\mathbf{A}) \mathrm{pH} 7.4$ and $(\mathbf{B}) \mathrm{pH} 5.0$.

Abbreviation: DOX, doxorubicin.

International Journal of Nanomedicine

\section{Publish your work in this journal}

The International Journal of Nanomedicine is an international, peerreviewed journal focusing on the application of nanotechnology in diagnostics, therapeutics, and drug delivery systems throughout the biomedical field. This journal is indexed on PubMed Central, MedLine, CAS, SciSearch $®$, Current Contents ${ }^{\circledR} /$ Clinical Medicine,

\section{Dovepress}

Journal Citation Reports/Science Edition, EMBase, Scopus and the Elsevier Bibliographic databases. The manuscript management system is completely online and includes a very quick and fair peer-review system, which is all easy to use. Visit http://www.dovepress.com/ testimonials.php to read real quotes from published authors. 